\title{
Zur morphembezogenen Überformung der deutschen Orthographie*
}

\author{
Nikolaus Ruge (Caen)
}

\begin{abstract}
Morphematic reorientation of German orthography takes place in conformity with a general law in the history of writing. Alphabetic writing systems, being necessarily phonographic, tend to develop towards the encoding of non-phonetic units. The emergence of morphematic elements in German is preceeded by the evolution of word-separation by regular spaces which had been adopted throughout Europe by the end of the $13^{\text {th }}$ century. Based on a corpus containing 157 High German texts (late $15^{\text {th }}$ to late $18^{\text {th }}$ centuries), this paper will demonstrate that morphematic reorientation of German orthography can be explained neither as an invisible-hand-process nor as the outcome of prescriptive grammar, but as the result of interaction between orthograpic norm and usage.
\end{abstract}

Three cases will be considered in detail:

(1) Graphic assimilation of allomorphic plosive variation emerges as early as the $12^{\text {th }}$ century, reflecting regional final devoicing. By the early $17^{\text {th }}$ century, the rule governing orthographic reprensentation of final devoicing in present-day German is fully adopted in usage. Its morphematic reinterpretation does not follow before the end of the $17^{\text {th }}$ century.

(2) Morphematic graphic representation of [a]-Umlaut emerges during the $14^{\text {th }}$ century in Upper Germany as a phonetic reflex of open [e]. It is recommended by Middle German grammarians since the 1560 s, with explicit mention of morphological factors. Around 1700 the writing rule imposes itself in usage.

(3) The use of double consonant letters occuring in final positions of 'graphic' syllables ( $<$ soll $>$ according to $<$ sollen $>$ ) rests inhibited until the $18^{\text {th }}$ century, in particular to prevent trior tessaragraphs (<sollt $>,<$ sollst $>$ ). It is the influence of Adelung's grammar which leads to the final adaption of the present-day rule.

\section{$1 \quad$ Einleitung}

Die morphematische Überformung der deutschen Orthographie gehorcht einem allgemeinen Gesetz der Schriftgeschichte, demzufolge Alphabetschriften im Laufe ihrer Evolution dazu tendieren, Bezüge zu nicht-phonischen Einheiten wie Morphemen oder Lexemen herzustellen. Abgesehen vom Sonderfall einer phonozentrischen Orthographiereform

\footnotetext{
* Für einige präzisierende und korrigierende Hinweise danke ich einem anonymen Gutachter.
} 
entwickeln sich flache so zu mehr oder weniger tiefen Schriftsystemen (cf. G. Sampson 1985). Wenn die Orthographie des Gegenwartsdeutschen mit P. Eisenberg (1996) als ein System von mittlerer Tiefe zu beschreiben ist, soll die vorliegende Untersuchung die sprachhistorischen Hintergründe dieser typologischen Charakteristik herausarbeiten.

Das Graphiesystem des Deutschen ist niemals in idealtypischem Sinne 'flach', also rein phonographisch gewesen, da sein graphisches Inventar dem Lateinischen entlehnt ist. Dies hat in der Frühzeit zu einer Reihe von Enkodierungsproblemen geführt, wie dem a. 863 Ad Liutbertum adressierten Brief Otfrids von Weißenburg zu entnehmen ist, auch wenn man diese Quelle, die stellenweise noch stark das geringe Prestige der Volkssprache gegenüber dem Lateinischen widerspiegelt, nicht überbewerten sollte (cf. R. Patzlaff 1975: 58).

Indessen war das lateinische Graphiesystem zum Zeitpunkt seiner Entlehnung in die deutschen Dialekte seinerseits dabei, Bezüge auf nicht-phonische Einheiten zu entwickeln, die aufgrund der noch lange währenden Abhängigkeit der Agenten volkssprachiger Schriftlichkeit von der lateinischen Schriftkultur quasi automatisch bei der graphischen Aufzeichnung der deutschen Dialekte eingesetzt werden konnten.

Notwendige Bedingung des Aufkommens morphembezogener Schreibungen ist dabei die Herausbildung der Worttrennung durch regelmäßige Spatien. Das mittelalterliche Abrücken von der scriptura continua beginnt im 8. Jahrhundert in englischen und irischen Skriptorien (cf. M.B. Parkes 1992: 23) und hat sich P. Saenger (1997) zufolge gegen Ende des 13. Jahrhunderts in ganz Europa durchgesetzt. In der lateinisch-althochdeutschen Schriftkultur ist nach B. Labs-Ehlert (1993: 180) die Worttrennung in volkssprachiger Überlieferung gleichwohl weniger konsequent als im Lateinischen. $\mathrm{Zu}$ den Denkmälern mit kaum entwickelter Worttrennung zählen u.a. das 'Georgslied' und die Wiener Otfrid-Handschrift ÖNB. 2687. Umgekehrt liegt mit der lateinisch-althochdeutschen Isidor-Bilingue eine frühe, umfangreiche Handschrift mit Worttrennung vor. Die konstitutive Zweisprachigkeit lateinisch-althochdeutscher Schriftlichkeit bedingt überdies nicht wenige schwer einzuordnende Einzelbeobachtungen. So alterniert in der Bilingue des 'Carmen Ad Deum' Latein Wort für Wort mit Althochdeutsch, Worttrennung durch Punkte kommt in den Segen 'Spurishalz' und 'Contra Vermes', im 'Ersten Straßburger Eid', in 'De Heinrico' und in der 'Würzburger Beichte' vor. Ausnahmslos durchgeführt ist die Worttrennung schließlich in sämtlichen Notker- und Williram-Handschriften.

Der Hinweis auf die Monsee-Wiener Bruchstücke des Codex ÖNB. 3043, wo der althochdeutsche Teiltext oft "Trennung der Silben eines Wortes" aufweist (B. Labs-Ehlert 1993: 114), ist im Zusammenhang der Enkodierung von Morphematischem von besonderem Interesse, weil hier offenbar erstmals mittels Spatien auf Einheiten oberhalb der Phonem- und unterhalb der Wortebene rekurriert wurde. Wie N. Henkel (1996: 59f.) am Beispiel der 'Murbacher Hymnen' der Handschrift Oxford, Bodleian Library Ms. Junius 25 zeigen konnte, handelt es sich allerdings um eine auf lateinisch-althochdeutsche Bilinguen beschränkte und nur im Rahmen von deren spezifischer Textualität zur Erhellung der Morphologie lateinischer Interpretamente funktionale Erscheinung. 
Wann aber treten im Deutschen Graphien auf, die ihrerseits als morphembezogen interpretiert werden können, und wann werden solche Graphien explizit als morphembezogen interpretiert?

$\mathrm{Zu}$ Klärung dieser Fragen wurden unter Zugrundelegung eines räumlich und zeitlich (15001770) gegliederten Korpus von 157 Textausschnitten von 4000 Wörtern ${ }^{1}$ drei Fälle, die in der Orthographie der deutschen Gegenwartssprache als morphemidentifizierende Schreibungen aufgefasst werden können (graphischer Ausgleich von Plosivalternanzen, Graphie der Umlaute von /a/ und /as/, Doppelkonsonantengraphie für die Silbenendrandpositionen einsilbiger Formen von sollen, wollen, können), auf ihre Entwicklung im Usus und ihre Behandlung in der zeitgenössischen grammatikographischen Norm hin untersucht.

\section{Der graphische Ausgleich der Plosivalternanzen}

\subsection{Das Aufkommen von Lenisbuchstaben in den Finalpositionen des graphischen Worts}

Die Lehr- und Handbuchmeinung, der zufolge die althochdeutschen stimmlosen Lenisplosive im Mittelhochdeutschen in Finalposition und vor stimmlosen Konsonanten zu stimmlosen Fortes 'verhärtet' wurden, um zum Neuhochdeutschen hin bei der graphischen Umsetzung der Inlautschreibung angepasst zu werden, hat sich schon seit längerem aus vielerlei Gründen als unhaltbar erwiesen. Ohne die Diskussion in ihrer Breite auch nur ansatzweise nachzeichnen zu können (dazu umfassend A. Mihm 2004), seien zwei wesentliche Punkte herausgehoben:

a) Die Fortisbuchstaben in den Finalpositionen des graphischen Worts (absoluter und gedeckter Auslaut) produzierende 'Auslautverhärtung' hat zwar stattgefunden, ist nach G.M. Vaught (1977) eher alt- als mittelhochdeutsch.

b) Die mittelhochdeutsche Periode ist demgegenüber durch zwei Lenisierungswellen charakterisiert, zum einen die binnendeutsche Konsonantenschwächung, zum anderen die mittelbairische Konsonantenschwächung, die ihrerseits möglicherweise in einer Reziprokrelation zur mittelhochdeutsch-frühneuhochdeutschen Apokopierung steht (cf. E. Kranzmayer 1956, F. Simmler 1983, P. Wiesinger 1996). Beide Entwicklungen produzieren Lenisbuchstaben in den Finalpositionen des graphischen Worts, die als Reflex dialektaler Lautungen $\mathrm{zu}$ betrachten sind. Der Anteil solcher oberflächlich mit den morphemidentifizierenden Schreibungen der Gegenwartssprache deckungsgleichen Graphien ist zu Beginn der neuhochdeutschen Periode schon erheblich. In der im 3. Viertel des 15. Jahrhunderts in Franken oder Hessen, also in jedem Fall in einem Lenisierungsgebiet aufgezeichneten Parzival-Handschrift Karlsruhe, Badische LB Cod. Donaueschingen 70 ist die Lenisbuchstabenschreibung für Dental zu 17,5\%, für Labial zu $40 \%$ und für Velar zu $69,5 \%$ durchgesetzt.

\footnotetext{
${ }^{1}$ Zitiert werden die Textausschnitte hier unter Angabe von Korpustext(=KT)-Nummer, Druckort und -jahr.
} 


\subsection{Determinanten der Durchsetzung von Lenisbuchstaben in den Finalpositionen des graphischen Worts nach 1500}

Am unteren zeitlichen Rand des hier zugrundeliegenden Korpus hat sich die Lage dahingehend vereindeutigt, dass Lenisbuchstabe 94\% der Labiale, 92,4\% der Velare und 67,4\% der Dentale in den einschlägigen Positionen repräsentiert. Allein im letztgenannten Fall verläuft der Alternanzausgleich also verhältnismäßig schleppend und erreicht erst bis 1620 annähernd sein gegenwartssprachliches Niveau, ohne dass dabei sprachlandschaftliche Faktoren eine Rolle spielen würden. In Fällen wie <tugent> oder <niemant> scheint die Verzögerung gleichwohl phonetisch, genauer: silbenstrukturell bedingt zu sein, liegt der Durchsetzungsgrad des Lenisbuchstaben für Nebensilbendental doch 1500 bei 43,6\%, um sich erst 1620 bei 93,7\% zu konsolidieren. Abgesehen davon blockieren nicht-phonische Faktoren die Durchsetzung von Lenisgraphien. $\mathrm{Zu}$ nennen ist einerseits die Verzögerung des Fortesabbaus in wortmedialer Position (cf. <leiplichen> [Leipzig 1498], <tôtlichen> [Straßburg 1510], <arckwenigs> [Nürnberg 1502]). Auffällig ist aber vor allem, dass die Verzögerungsfälle nicht gleichmäßig über das Lexikon verteilt sind: 770 der einschlägigen Graphien (tokens) entfallen auf 13 types, während sich die übrigen 238 tokens auf 85 types verteilen. Die Verzögerung bei den so ermittelten zentralen Fällen lässt sich vielfach auf morphologische bzw. morphosemantische Faktoren zurückführen, deren Wirkung sich allerdings z.T. mit den Effekten silbenstruktureller (tugent, iugent, tausent, niemant/iemant) bzw. positioneller Faktoren überschneidet (bei Graphien wie <tugentreich>). Im Einzelnen lassen sich unterscheiden:

a) Die synchrone Konkurrenz archaischer Formen: <magt $>/<\operatorname{magd}>$ vs. $<$ maget $>$, $<($ n)iemand/(n)iemant $>$ vs. $<($ n)ieman $>,<$ wird $>/<$ wirt $>$ vs. $<$ wirdet $>$

So konkurrieren etwa im Ind. Sg. Präs. von werden während der Übergangsperiode vom Mittelhochdeutschen zum Neuhochdeutschen zwei Paradigmen, die Explizitform <wirdet> und die Kontraktionsform <wirt>, später <wirdt> bzw. <wird>. Für die genauen Umstände der Durchsetzung variieren die Angaben der Handbücher (cf ${ }^{1}$ DWB. XI.1.2, Sp. 223; U. Dammers/W. Hoffmann/H.-J. Solms 1988: § 64,2), die vollständige Durchsetzung der Kontraktionsform erscheint aber in jedem Fall als notwendige Bedingung der Lenisgraphie, die bei Luther a. 1528 durchgesetzt ist (cf. H. Bach 1985: 168f.).

Die Abhängigkeit der Durchsetzung der Lenisschreibung vom Verschwinden der Vollform $<$ wirdet $>$ ist denn auch an ihrem Vorkommen im Textkorpus exemplarisch nachzuvollziehen. Die Konkurrenz zwischen den Paradigmen verliert kontinuierlich an Bedeutung, herrscht aber noch bis einschließlich 1560. Die letzten Schreibungen von wird mit Fortisbuchstaben finden sich entsprechend in KT 70 [Mainz 1595]. Bis 1650 konkurrieren Lenis- und Kompromissgraphie, bis auch letztere abgebaut ist: 


\begin{tabular}{|l|l|l|l|l|}
\hline Zeitschnitt & <wirdet> & $<$ wirt & $<$ wirdt & $<$ wird \\
\hline 1500 & $19,7 \%$ & $71,4 \%$ & $8,4 \%$ & $0,6 \%$ \\
\hline 1530 & $11,7 \%$ & $26,4 \%$ & $7,9 \%$ & $53,9 \%$ \\
\hline 1560 & $0,9 \%$ & $53,1 \%$ & $23,2 \%$ & $22,8 \%$ \\
\hline 1590 & $0 \%$ & $12,6 \%$ & $28,2 \%$ & $59,2 \%$ \\
\hline 1620 & $0 \%$ & $0 \%$ & $42,1 \%$ & $57,9 \%$ \\
\hline 1650 & $0 \%$ & $0 \%$ & $6,8 \%$ & $92,2 \%$ \\
\hline 1680 & $0 \%$ & $0 \%$ & $0 \%$ & $100 \%$ \\
\hline
\end{tabular}

Tabelle 1: Die Graphien für die 3. Sg. Ind. Präs. von werden 1500-1680

b) Das Fehlen expliziter Flexionsformen als Konsequenz des Umbaus des Flexionssystems: $<$ hand $>/<$ hant $>$, $<$ kind $>/<$ kint $>$

Bei der Pluralmarkierung von Hand wechseln während des gesamten Untersuchungszeitraums besonders in Apokopierungsregionen völlig ungeregelt $-e$ und Nullendung (cf. K.-P. Wegera 1987: §81). Die hier untersuchten Texte weisen dafür zahlreiche Belege auf, wenn sich die Relation Nullendung : Plural-e auch nach 1530 zugunsten des letztgenannten entwickelt: ZS 1 (1500) 2 : 0 - ZS 2 (1530) $7: 3$ - ZS 3 (1560) 7 : 0 - ZS 4 (1590) $1: 4$ - ZS 5 (1620) $1: 4$ - ZS 6 (1650) $0: 3$ - ZS 7 (1680) $0: 7$ - ZS 8 (1710) 4 : 2. Diese Varianz erschwert, wie im vergleichbaren Fall Kind, den morphemidentifizierenden Bezug auf Explizitschreibungen (cf. $<$ Hände $>/<$ Hand $>$, $<$ Kinder $>/<$ Kind $>$ in den Gegenwartssprache).

c) Die Neutralisierung der Numeruskategorie: $\quad<$ gold $>\mid<$ golt $>, \quad\langle$ pfund $>|<$ pfunt $>$, $<$ tausend $>\mid<$ tausent $>$, <iugend $>/<$ iugent $>$

Gold ist ein Kontinuativum, für das wie in den vergleichbaren Fällen Pfund und tausend (cf. M. Walch/S. Häckel 1988: § 144 Anm. 11) die Realisierung des semantischen Konzeptes Pluralität durch die morphologische Kategorie Numerus schwer vorstellbar ist (*zwei Golde vs. zwei Unzen Gold). Im Korpus findet sich konsequenterweise keine Pluralform, deren graphische Umsetzung als Orientierungspunkt eines morphematisch motivierten Ersatzes von $<$ golt $>$ durch < gold $>$ hätte dienen können. Die unabhängig davon mögliche Orientierung an den Flexionsformen des Singular wird darüber hinaus zu Beginn des Untersuchungszeitraums von graphischen Reflexen auf konkurrierende Aussprachevarianten überlagert. In KT 12 [Erfurt 1499] entspricht der Varianz der Nominativgraphien <golt> [p. 3 u. 11] bzw. <gold> [p. 2] die Varianz der Genitivgraphien <goldes> [p. 4] bzw. <goltes> [p. 9].

Unter den statistisch ermittelten zentralen Fällen verzögerter Durchsetzung der Lenisschreibung entziehen sich nur zwei Lexeme einer morphologischen bzw. morphosemantischen Erklärung ihrer konservativen Graphie. Dabei handelt es sich einerseits um $<$ geld $>/<$ gelt $>$, dessen Graphie sowohl durch das Nominalparadigma als auch durch das Verb gelten motivierbar ist, andererseits um $<$ gedult $>/<$ geduld $>$, dessen Flexionsparadigma nach Ausweis der Korpustexte ausschließlich Fortisgraphien aufweist,. 


\subsection{Die Graphie $<\mathrm{dt}>$}

In einer Übergangsphase bis etwa 1560 führt der Abbau der Fortisgraphien für Dental nicht automatisch $\mathrm{zu}$ einem linearen Anstieg der Lenisgraphien, sondern zieht eine zwischenzeitliche Konjunktur der so genannten Kompromissgraphie $<\mathrm{dt}>$ nach sich:

\begin{tabular}{|l|l|l|l|}
\hline Zeitschnitt & $\langle\mathrm{d}\rangle$ & $\langle\mathrm{dt}\rangle$ & $<\mathrm{t}\rangle$ \\
\hline 1500 & $58,4 \%$ & $9,2 \%$ & $32,4 \%$ \\
\hline 1530 & $70,59 \%$ & $12,3 \%$ & $11,2 \%$ \\
\hline 1560 & $57,2 \%$ & $25,1 \%$ & $11,7 \%$ \\
\hline 1590 & $73,4 \%$ & $13,9 \%$ & $12,7 \%$ \\
\hline 1620 & $91,5 \%$ & $6,7 \%$ & $1,9 \%$ \\
\hline 1650 & $85,9 \%$ & $12,3 \%$ & $1,7 \%$ \\
\hline 1680 & $96,7 \%$ & $2,9 \%$ & $0,3 \%$ \\
\hline 1710 & $98,4 \%$ & $1,4 \%$ & $0,2 \%$ \\
\hline
\end{tabular}

Tabelle 2: Verhältnis von $<\mathrm{d}>,<\mathrm{dt}>$ und $<\mathrm{t}>1500-1710$

Wie der Übersicht entnommen werden kann, sind bis 1710 bei weitem nicht alle <dt>Graphien abgebaut. Ein Einzelfall sticht sowohl bei der Auswertung der Handbücher als auch bei der Korpusanalyse besonders heraus: Die graphische Umsetzung der hinsichtlich ihrer Zuordnung zum Substantiv- oder Adjektivparadigma zweideutigen Silbe [tort], die auffällig häufig und noch weit bis ins Neuhochdeutsche durch Rückgriff auf die <dt>-Graphie gelöst wurde (cf. H. Bach (1985: § 148.5); E. Glaser (1985: §199) und V. Moser (1929: § 29.4)). Im letzten Zeitschnitt (1710) kommen noch $17<\mathrm{dt}>-$-Schreibungen gegen die gegenwärtige Norm vor. Neben $<$ Begierdt $>$ [Regensburg 1710] und <tausendt $>$ [Straßburg 1711] handelt es sich dabei in erster Linie um:

<Todt> [Hamburg 1687]; <Todt> [Augsburg 1706]; <todt> [Augsburg 1706]; <Todt> [Straßburg 1712]; <todt> [Straßburg 1712]; <Todt> [Coburg 1713]; <Todt=Feinden> [Leipzig 1711]; <Todt> [Köln 1710]; <Todt> [Köln 1712]; <todtschwachen> [Köln 1712]

Die Aufgabe der Kompromissgraphien <todt> bzw. <tödten> gehört auf der I. Orthographischen Konferenz a. 1876 zu den Änderungsvorschlägen Rudolf von Raumers (cf. D. Nerius et al. ( ${ }^{3} 2000$ : 333)), deren Realisierung in der Gegenwartssprache zu einer willkürlichen ${ }^{2}$ Regelung der Standardaussprache und der darauf beruhenden Schreibungen $<$ Tod $>$ [to:t] wegen $<$ Tode $>$ ['to:də] gegenüber $<$ tot $>$ [tort] wg. $<$ tote $>$ [tortə] geführt hat.

\subsection{Die Reflexion des Usus in der zeitgenössischen Grammatikographie}

Hinsichtlich der Herausbildung der historischen Norm darf aus der Tatsache, dass bei der endgültigen Durchsetzung der Lenisschreibungen augenscheinlich auch morphologische Faktoren eine Rolle spielten, noch nicht auf das Wirken eines wie auch immer gearteten morphematischen Schreibprinzips geschlossen werden. Zur Ergänzung der vorliegenden ausdrucksseitigen Indizien bedürfte es zusätzlich zeitgenössischer metasprachlicher Quellen,

\footnotetext{
${ }^{2}$ Zu Inkonsequenzen, die sich daraus für die gegenwärtige Orthographie ergeben haben cf. M. Kohrt (1991: 537 Fn 8).
} 
die eine solche Motivation explizieren würden oder doch entsprechend interpretierbar wären. Hinsichtlich der Regelung der Auslautalternanzen ist dies erstmals in der 'Grammatica germanicae linguae' (1578) von J. Claius der Fall (cf. Moulin 2004: 49ff.), zu einem Zeitpunkt also, als die Durchsetzung im Usus bereits weit fortgeschritten scheint. Claius' explizit morphologische Begründung - "In his spectanda est deriuatio, \& numerus pluralis, vt: Das Kalb / Vitulus. non Kalp / quia plurale est Die Kelber" - ist demnach als Remotivation einer ursprünglich phonetisch motivierten und im Usus bereits weitgehend konsolidierten Graphie aufzufassen.

\section{Die graphische Repräsentation der Umlaute von /a/ und /as/}

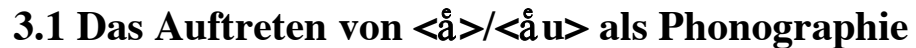

Der statistischen Tatsache, dass die überwiegende Mehrzahl der Okkurrenzen der gegenwartssprachlichen Graphien <ä> für $/ \varepsilon /$ bzw. <äu> für $/ \supset e^{/ 3}$ als morphembezogene Schreibungen interpretiert werden können ( $<$ Hände $>$ wegen $<$ Hand $>$, $<$ bläulich $>$ wegen $<$ blau $>$ ) steht die Existenz von Schreibungen gegenüber, bei denen das Auftreten von $<a ̈>/<a ̈ u>$ synchron nicht mehr morphematisch motiviert ist (<dämpfen $>$ als Kausativum zu fnhd. dimpfen 'dampfen', <grässlich> zum im 19. Jh. veralteten Adj. graß 'wütend, zornig') bzw. diachron nie morphematisch motiviert war (<Säule $>)$.

Betrachtet man frühneuhochdeutsche Texte, vervielfachen sich solche Fälle. Für Schreibungen wie <Båcher> [KT 98, Straßburg 1647], <Båttler> [KT 77, Basel 1624], $<$ Thråschl> [zu dreschen, KT 21: Regensburg 1533], <ărtz> 'Erz' [KT 41: Basel 1559], <fåldt> 'Feld' [KT 42: Basel 1557], <gålb> [KT 61: Straßburg 1588], <håfftig> [KT 61: Straßburg 1588], <kåck> [KT 22: Augsburg 1532], <abmåssen> [KT 99: Nürnberg 1653], <schlåchten> 'schlichten' (Adj.) [KT 42: Basel 1557], <zerschmåttert> [KT 41: Basel 1559], <wårt> 'wert' [KT 42: Basel 1558] oder <wåtter> [KT 77: Basel 1624] lassen sich, selbst bei (in diesem Zusammenhang zwangsläufig fiktivem) Rückgang auf die germanischen Lautverhältnisse, keine Anknüpfungspunkte für eine morphematische Motivation herstellen.

Solche nicht zufällig durchweg oberdeutschen Druckorten entstammende Schreibungen bilden den späten Reflex des Umstandes, dass die Graphie <å > für Kurzvokal zum Zeitpunkt ihres Auftretens im 12. Jahrhundert (cf. H. Paul ${ }^{24} 1998$ : § 63) als Bezeichnung des durch Sekundärumlaut entstandenen $e$-Kurzvokals mit dem jeweils größten Öffnungsgrad im Bairischen, Schwäbischen und Hochalemannischen phonetisch motiviert ist (cf. V. Moser (1929: § 17)4. Gegen Ende des 15. Jahrhunderts ändert sich die Situation zunächst bezüglich

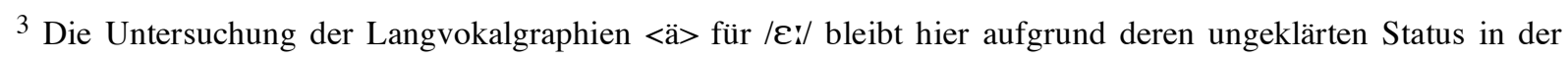
Gegenwartssprache ausgeschlossen

${ }^{4}$ Diese Beobachtung betrifft das Aufkommen der Graphie. Neuere Überblicksdarstellungen bei R.P. Ebert et al. (1993: § L12) und speziell bei K.-P. Wegera (1987) liefern hinsichtlich ihrer Durchsetzung reichlich Hinweise auf eine spätere Dominanz von $<\mathrm{e}>$ in den oberdeutschen Schreibsprachlandschaften. Nach der auf die Pluralmarkierung beschränkten Darstellung von K.-P. Wegera (1987: 225f.), die allerdings nicht auf die Motivation rekurriert, überwiegt die <å>-Graphie (mittel)bairisch erst im späten 16. Jahrhundert und setzt sich im 17. Jahrhundert auf Normniveau durch. Westoberdeutsch dominiert <e> noch in Ulm 1486 und erreicht dort 1660 Normniveau (cf. K.-P. Wegera 1987: 229), während <å> in Straßburger Drucken überhaupt erst im 16.
} 
der räumlichen Ausdehnung, als das Zeichen $<\grave{a}>$ in nichtoberdeutschen Sprachlandschaften auftritt. Für die Erklärung der genauen Umstände konkurrieren zwei Sichtweisen. Während R. Hotzenköcherle (1962: 322) funktional argumentiert und keine phonetische Motivation für md. <å > in Betracht zieht, betont V. Moser (1929: §70,1) eher den graduellen Charakter des Eindringens von <å̀ ins Mitteldeutsche und setzt zumindest für das Rheinfränkische in Einzelfällen noch phonisch induzierte <å $>$ an, als deren Ursache beispielsweise Schreibaussprachen anzusetzen wären. Grundsätzlich aber bleibt festzuhalten:

a) <å> für Kurzvokal tritt seit dem späten 15. Jahrhundert zunehmend in Schreiblandschaften auf, wo ihm kein phonisches Pendant entspricht. Westmitteldeutsch bzw. rheinfränkisch überwiegt nach V. Moser (1929: § 70.1) von Anfang an und verstärkt nach 1550 der Gebrauch für morphematisch flankierten (Sekundär)Umlaut, abgesehen v.a. von Frankfurter Drucken. Vergleichbare Verhältnisse liegen nach 1570 in ostmitteldeutschen und

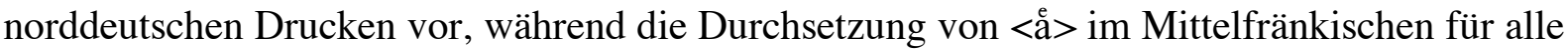
Herkunftsfälle bis in die ersten Jahrzehnte des 17. Jahrhunderts zögerlich bleibt. ${ }^{5}$

b) In Sprachlandschaften, die phonetisch motiviertes <å > aufweisen, verschwindet es zunehmend in Fällen, wo keine morphematische Flankierung vorliegt. Bairisch ist das erst im 17. Jahrhundert der Fall, im Schwäbischen bereits nach 1550, während morphematisch isoliertes <å> für Kurzvokal im Hochalemannischen zwar ebenfalls im 17. Jahrhundert zurückgeht, jedoch in dialektal bedingt geringerem Maße. Ähnliches gilt für niederalemannische Drucke (cf. V. Moser 1929: 120).

Auch die Diphthongschreibung <åu> tritt zunächst als Phonographie auf. Bairisch und ostschwäbisch dient sie gleichermaßen zur Bezeichnung der Produkte der neuhochdeutschen Nukleussenkung von mhd. öu (vröude; böume) wie der Diphthongierung des mhd. Umlaut-iu (hiuser) gegenüber dem weniger offen artikulierten diphthongierten mhd. iи (niuwe). Ein eindeutiges Indiz für die Aufgabe der phonetischen Motivation von <åu > ist in der bairischen Durchsetzung von <eu> für nicht umgelautetes mhd. iu (niuwe) im 15. Jahrhundert zu sehen (cf. V. Moser 1929: 188). Dennoch schwankt der Gebrauch hier mit abnehmender Tendenz bis ins 17. Jahrhundert, wovon eine Schreibung wie <stråye> zu mhd. ströuwen [KT 76: Wien 1625] zeugt.

Alemannisch setzt sich morphematisch flankiertes <åu> vor 1550, niederalemannisch um 1600 durch (cf. V. Moser 1929: § 79.I.2). Phonetische Motivierung kommt hier nie in Frage, da morphematisch flankiertes <åu> den durch Sekundärumlaut entstandenen mhd. Diphthong $\ddot{o u}$ bezeichnet (zur unterbliebenen Nukleussenkung cf. H. Paul ${ }^{24} 1998$ : § 44), der sich alemannisch in manchen Drucken bis ins 17. Jahrhundert hält (cf. V. Moser 1929: § 79.I.2).

Grundsätzlich verschieden ist die Situation auch im Mitteldeutschen, Oberfränkischen und in Nürnberg, wo mhd. ei, ou, öu durchgehend spätestens im 13. Jahrhundert monophthongiert

\footnotetext{
Jahrhundert auftritt (cf. K.-P. Wegera 1987: 231), um relativ rasch (nach a. 1590) dominant zu werden, in Zürich schließlich halten sich <å $>$ und <e> noch a. 1578 die Waage (cf. K.-P. Wegera 1987: 233).

${ }^{5}$ Nach der auf die Pluralmarkierung beschränkten Darstellung bei K.-P. Wegera (1987: 237-241) finden sich die ersten hessischen Belege im späten 15. Jahrhundert, während in einzelnen ripuarischen Drucken $<\grave{\mathrm{a}}>$ auch am Ende des 17. Jahrhunderts noch peripher erscheint, wohingegen omd. <å̀ im 16. Jahrhundert eindringt.
} 
werden $^{6},<$ åu >-Schreibungen also seitdem die phonische Grundlage fehlt. Wo hier - von der späten Ausnahme <låugnen > abgesehen $-<$ åu > auftritt, kann nur morphematische Motivation vorliegen.

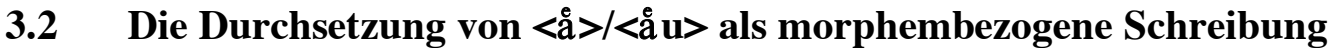

Der Korpusbefund bestätigt und akzentuiert die Aussagen der vorgängigen Forschung insbesondere dahingehend, dass es sich bei der Durchsetzung als morphembezogen

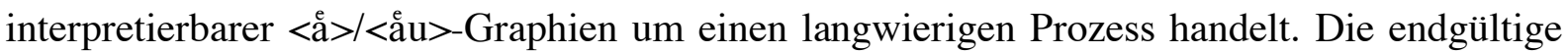

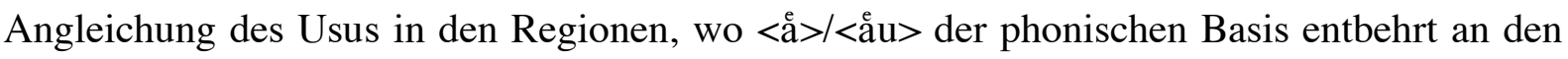
Usus im Oberdeutschen und Westmitteldeutschen lässt sich erst Anfang des 18. Jahrhunderts konstatieren, wobei das Nordoberdeutsche eine vermittelnde Stellung einnimmt. Kontrastiert man die Befunde zum Oberdeutschen mit den Befunden zum Mittel- und Norddeutschen, ergibt sich folgendes Bild:

\begin{tabular}{|l|l|l|}
\hline Zeitschnitt & $\begin{array}{l}\text { phonische Basis für } \\
<\grave{a}>(O b d .)\end{array}$ & $\begin{array}{l}\text { keine phonische Basis } \\
\text { für <å }>\text { (Md., Norddt.) }\end{array}$ \\
\hline 1500 & $10,4 \%$ & - \\
\hline 1530 & $14,7 \%$ & - \\
\hline 1560 & $29,7 \%$ & - \\
\hline 1590 & $37,1 \%$ & $7 \%$ \\
\hline 1620 & $77,7 \%$ & $58 \%$ \\
\hline 1650 & $80,5 \%$ & $70 \%$ \\
\hline 1680 & $95,2 \%$ & $78 \%$ \\
\hline 1710 & $97,6 \%$ & $97 \%$ \\
\hline
\end{tabular}

Tabelle 3: Durchsetzung von <å $>$ in Sprachlandschaften mit und ohne phonische Basis

Die Überwindung sprachlandschaftsspezifischer Schreibgewohnheiten lässt sich beispielhaft

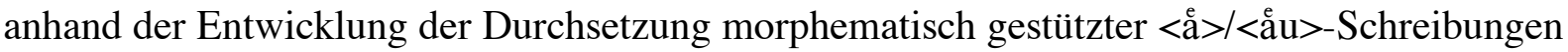
während des Zeitintervalls 1590-1620 verdeutlichen :

Vor 1620 sind für den Verlauf der Durchsetzung bei den oberdeutschen Korpustexten Diskontinuitäten prägend: Dies gilt für das Ostoberdeutsche grundsätzlich bis 1590, für das Westoberdeutsche für das Intervall 1560 bis 1590. Danach gewinnt die Durchsetzung, zunehmend lautungsunabhängig, an Kontinuität und wird in ihrer Tendenz unumkehrbar. Eine Vorreiterrolle nehmen hier die westmitteldeutschen Drucke ein, wo der Anteil an $<\mathrm{a}>/<a ̊ u ~>-$ Schreibungen von 14,04\% (1530) über 34,07\% (1560), 44,48\% (1590) auf 82,61\% (1620) steigt. 1650 ist gleichwohl ein letzter, regional begründeter Rückgang auf $71,90 \% \mathrm{zu}$ verzeichnen ${ }^{7}$, bis die <å $><$ <åu $>$-Schreibungen 1680 (95,87\%) und 1710 (98,01\%) das Niveau der gegenwärtigen orthographischen Norm erreichen.

\footnotetext{
${ }^{6}$ Zu den genaueren Umständen cf. V. Moser (1929: § 79.II.2), K.-P. Wegera (1987: 231, 237, 241).

7 Dieser Rückgang reflektiert die schon von V. Moser (1929: § 70.1) bemerkte, auf Grund der hier gewählten Korpusstruktur aber nicht auf den ersten Blick erkennbare Verzögerung mittelfränkischer Drucke. Alle westmitteldeutschen Korpustexte des Zeitschnitts 1620 stammen aus Frankfurt (Main), diejenigen des fraglichen
} 
Eine bruchlose Kontinuität zeigen dann die Korpustexte der nordoberdeutschen

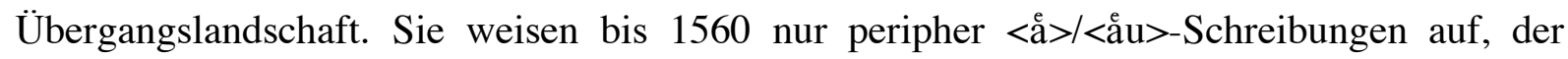
entscheidende Sprung findet hier zwischen 1560 und 1590 statt, wo sich die relative Durchsetzung um den Faktor 6,75 von 2,86\% auf 19,32\% steigert. Abgesehen von einem späten, vernachlässigbaren Absinken ist ab diesem Zeitpunkt eine kontinuierliche Durchsetzung bis auf $100 \%$ (ZS 1680) zu beobachten.

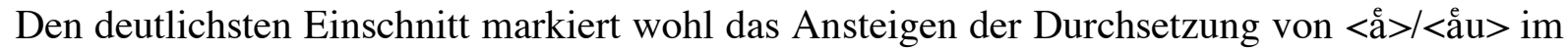
Ostmitteldeutschen um den Faktor 7,91 von 1590 (7,35\%) bis 1620 (58,19\%). Bis

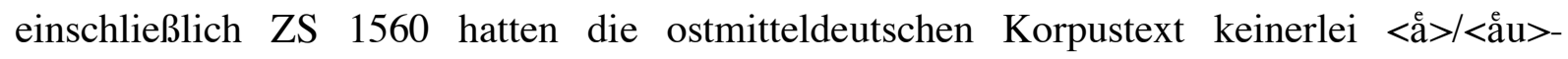
Schreibungen aufgewiesen, die weitere Steigerung bis auf Normniveau (1710 mit 98,51\%) verläuft wesentlich schleppender.

Die mit 42,88\% relativ hohe Einstiegsdurchsetzung in KT 107 [Schleswig 1640] schließlich erklärt sich nicht nur durch das Fehlen einer phonischen Basis, sondern weist auch darauf hin, dass die norddeutschen Drucke bereits auf eine relativ konsolidierte Tradition morphematisch motivierter $<\mathfrak{a}>\mid<a ̊ u>-S c h r e i b u n g$ zurückgreifen können.

Unterhalb der Sprachlandschaftsebene haben morphologische Faktoren gleichwohl den Verlauf der Durchsetzung in den untersuchten ostmitteldeutschen Drucken beeinflusst. Beim

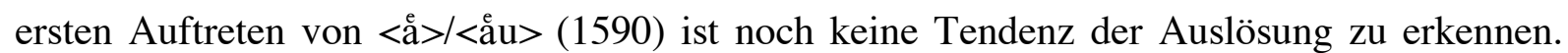
Zwar sind in KT 67 [Leipzig 1592] alle <å>-Schreibungen durch Flexion motiviert ${ }^{8}$, in KT 65 [Erfurt 1589] löst demgegenüber ausschließlich das Formationsmorphem -ig (wenige) $<$ à $>/<a ̊ u l>-S c h r e i b u n g e n$ aus $^{9}$. Die im Befund zu KT 67 angelegte Tendenz vereindeutigt sich zum ZS 1620. Im Bereich der flexionsausgelösten Schreibungen dominiert die Substantivflexion, wie sich exemplarisch für den Befund der ostmitteldeutschen Texte des Zeitschnitts 1620 lässt (für die einzelnen Auslösungsfälle sind die absoluten Zahlen angegeben):

\begin{tabular}{|l|l|l|l|l|l|}
\hline KT & $\begin{array}{l}\text { Durchsetzung } \\
\text { gesamt }\end{array}$ & $\begin{array}{l}\text { Durchsetzung } \\
\text { Flexion }\end{array}$ & $\begin{array}{l}\text { Adjektiv } \\
\langle\mathrm{a}\rangle /\langle\mathrm{e}\rangle\end{array}$ & $\begin{array}{l}\text { Substantiv } \\
\langle\mathrm{e}\rangle /<\mathrm{e}\rangle\end{array}$ & $\begin{array}{l}\text { Verb } \\
\langle\mathrm{e}\rangle /<\mathrm{e}\rangle\end{array}$ \\
\hline 83 (Wittenberg 1619) & $42,55 \%$ & $72,73 \%$ & $1 / 1$ & $15 / 3$ & $0 / 2$ \\
\hline 84 (Leipzig 1620) & $73,42 \%$ & $66,67 \%$ & $3 / 2$ & $22 / 0$ & $0 / 2$ \\
\hline 85 (Wittenberg 1621) & $49,02 \%$ & $50 \%$ & $0 / 2$ & $4 / 0$ & $2 / 3$ \\
\hline
\end{tabular}

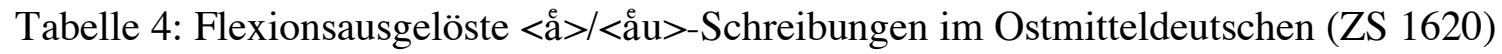

Bei derivationsausgelösten Schreibungen liegen zwar gleichfalls hohe Durchsetzungsraten vor, von KT zu KT ist aber noch mit großen Divergenzen zu rechnen, sowohl auf Gesamttextebene als auch hinsichtlich der betroffenen Wortart:

\footnotetext{
Zeitschnitts 1650 sämtlich aus Köln. Zeitschnitt 1680 enthält keine Kölner Drucke, zum Zeitschnitt 1710 erreichen dann alle untersuchten Drucke Normniveau.

8 <Gårten> [p. 36], <Bienengårten> [p. 36], <Båchen> [p. 36], <Rotbårte> [p. 33], daneben aber noch die Pluralgraphie $<$ Feuste $>$ [p. 25].

$9<$ beståndige $>$ [f. A $v^{v}$ ], <Glåubigen $>$ [2x f. A $A^{v}$ ], daneben aber <einfeltigst> [f. A $v^{r}$ ], <einfeltig> [f. B $2^{r}$ ], $<$ gewertig $>$ [f. C $8^{v}$ ], <andechtigem $>$ [f. $\mathrm{B}^{\mathrm{r}}$ ], <unbendige $>$ [f. C $8^{\mathrm{r}}$ ].
} 


\begin{tabular}{|l|l|l|l|l|l|}
\hline KT & $\begin{array}{l}\text { Durch- } \\
\text { setzung } \\
\text { gesamt }\end{array}$ & $\begin{array}{l}\text { Durchsetzung } \\
\text { Derivation }\end{array}$ & $\begin{array}{l}\text { Adjektiv } \\
<\mathrm{e}>/<\mathrm{e}>\end{array}$ & $\begin{array}{l}\text { Substantiv } \\
<\mathrm{a}>/<\mathrm{e}>\end{array}$ & Verb $<\mathrm{a}>/<\mathrm{e}>$ \\
\hline 83 & $42,55 \%$ & $16 \%$ & $2 / 11$ & $1 / 8$ & $1 / 2$ \\
\hline 84 & $73,42 \%$ & $80 \%$ & $11 / 4$ & $4 / 0$ & $17 / 4$ \\
\hline 85 & $49,02 \%$ & $48,78 \%$ & $16 / 16$ & $2 / 2$ & $1 / 3$ \\
\hline
\end{tabular}

Tabelle 5: Derivationausgelöste $<\grave{e}>/<$ åu $>-$ Schreibungen im Ostmitteldeutschen (ZS 1620)

\subsection{Norm und Usus}

Betrachtet man das Verhältnis Usus/Norm, so lässt sich zunächst konstatieren, dass $<\mathrm{a}>/<\mathrm{a} u>$, ähnlich wie dies beim graphischen Ausgleich der Plosivalternanzen der Fall war, als Reflex dialektaler Lautung auftreten. Ihre Durchsetzung ist gleichwohl zum Zeitpunkt des Einsetzens grammatischer Reflexion über die Volkssprache keineswegs in vergleichbarem Maße konsolidiert. Je nach sprachlandschaftlicher Verortung der Akteure sind überdies Differenzierungen erforderlich (cf. C. Moulin 2004: 44ff.). Wenn der Oberdeutsche J. Kolross 1530 Umlautgraphie für Derivationsprodukte vorschreibt, handelt es sich um eine morphologische Reinterpretation einer phonographisch bis dahin kaum reflektierten und damit funktionalisierbaren Graphie, die sich danach im Westoberdeutschen zwar nicht kontinuierlich, aber am Ende doch durchsetzt (s.o.), hinsichtlich deren morphematischer Motivation jedoch Zweifel angebracht sind.

Die ein Jahr später vom Ostmitteldeutschen F. Frangk formulierte, oberflächlich betrachtet mit Kolross' Vorschrift äquivalente Schreibregel ist hingegen, berücksichtigt man die Herkunft ihres Urhebers, als rein morphembezogen aufzufassen, wenn auch eine Kenntnis der Herkunft des Zeichens <å> als Reflex oberdeutscher Aussprache und damit die Diagnose Reinterpretation nicht kategorisch auszuschließen ist. Im Unterschied zur Situation bei Kolross ist aber festzustellen, dass die frangksche Regel keinen unmittelbaren Einfluss auf den regionalen Graphieusus zeitigt. Die ersten ostmitteldeutschen <å >-Graphien erscheinen, wie oben herausgearbeitet wurde, erst mehr als ein halbes Jahrhundert nach der Formulierung der explizit morphematischen Schreibregel.

\section{Doppelkonsonantenbuchstabe (DKB) für die Silbenendrandpositionen einsilbiger Formen von sollen, wollen, können}

\subsection{Funktion und Aufkommen}

Ein wiederum anders gelagertes Zusammenspiel von Norm und Usus bei der Durchsetzung einer morphemidentifizierenden Schreibregel ist hinsichtlich der DKB-Schreibung in den Silbenendrandpositionen einsilbiger Formen der neuhochdeutschen Modalverben sollen, wollen und können beobachtbar, die hier exemplarisch für diesen Regelbereich behandelt werden sollen. Ihre morphembezogene Interpretation ist für die Gegenwartssprache nicht unumstritten und bedarf der Erläuterung. Dem akzentbasierten Ansatz folgend wäre eine Schreibung wie <kann> phonographisch, <kannst> aber als morphembezogen zu interpretieren (cf. K.H. Ramers 1999: 53ff.), dem silbenbasierten Ansatz zufolge sind <kann> 
wie $<$ kannst $>$ gleichermaßen dadurch zu erklären, dass "der entsprechende Konsonant in einer anderen Form des Paradigmas Silbengelenk ist" (P. Eisenberg 1998: 68f.). Während einzelne Graphien der gegenwärtigen Norm den silbenbasierten Ansatz in Erklärungsnot bringen (<hat $>$ trotz <hatten $>$, <-in $>$ trotz <-innen $>$, legt die diachrone Perspektive nahe, zur Erklärung der hier in Frage stehenden Schreibungen dennoch für letzteren zu plädieren. Die Schreibgeminaten, die in der Gegenwartssprache das Silbengelenk repräsentieren, unterscheiden sich zwar in ihrer Herkunft (für können ist diese unklar (cf. E. Seebold 1966: 282), für wollen handelt es sich um die westgermanische Konsonatengemination, für sollen um Analogie im Mittelhochdeutschen), sind aber im Mittelhochdeutschen insofern funktional äquivalent, als sie phonische Geminaten repräsentieren, denen in den Silbenendrandpositionen ihrer Flexionsparadigmen durch Einzelbuchstaben wiedergegebene Einzellaute gegenüberstehen - phonische Gemination ist dort nur vormittelhochdeutsch für den Nasal wahrscheinlich zu machen. Anders als in den beiden bis hierher behandelten Fällen liegt damit keine morphematische Reinterpretation einer Phonographie vor.

\subsection{DKB-Schreibungen im Frühneuhochdeutschen: Graphematische Anomie und inhibitorische Faktoren}

Der Umstand, dass der DKB trotz des Fehlens einer denkbaren phonetischen Motivation schon früh in den Silbenendrandpositionen auftritt - weniger für den Nasal, wo nach dem durch den Korpusbefund weitgehend bestätigten Urteil der einschlägigen Untersuchungen (cf. Ebert et al. 1993 § L62,2; E. Otto 1970: 244, H. Boková 1998: 301, H. Bach 1985: 367) noch lange die im Mhd. vorherrschende Verteilung dominiert, als für den Labial - hat die Forschung bisweilen veranlasst, eine Art graphematischer Anomie zu konstatieren:

"Das für die Graphie des /1/ typische Nebeneinander von $l$ und $l l$ ist medial vorkonsonantisch und final nachvokalisch größtenteils fakultativ (...). Seit der 2. Hälfte des 15 . Jhs. stehen $l$ und $l l$ ohne erkennbare Regel nebeneinander, und zwar unabhängig davon, ob ein Kurzvokal oder ein Langvokal bzw. Diphthong vorangeht" (Ebert et al. 1993 § L64)

Auf der diachronen Makroebene lässt sich dieser Befund zunächst durchaus bestätigen. Die Entwicklung im Usus ist für alle drei untersuchten Verben sprachlandschaftsunabhängig teilweise diskontinuierlich, teilweise stagnierend, einziger gemeinsamer Nenner ist die späte Konsolidierung der gegenwärtigen Norm um 1770: 


\begin{tabular}{|l|l|l|l|}
\hline Zeitschnitt & Sollen & wollen & können \\
\hline 1500 & $15,3 \%$ & $19,7 \%$ & $0 \%$ \\
\hline 1530 & $44,8 \%$ & $20 \%$ & $0 \%$ \\
\hline 1560 & $24,9 \%$ & $22,6 \%$ & $6,6 \%$ \\
\hline 1590 & $27,1 \%$ & $17,7 \%$ & $0 \%$ \\
\hline 1620 & $28,1 \%$ & $24,3 \%$ & $0 \%$ \\
\hline 1650 & $31,2 \%$ & $25,8 \%$ & $2 \%$ \\
\hline 1680 & 62,5 & $38,5 \%$ & $4,5 \%$ \\
\hline 1710 & $61,4 \%$ & $50,3 \%$ & $5,8 \%$ \\
\hline 1740 & $67,8 \%$ & $74,3 \%$ & $31,9 \%$ \\
\hline 1770 & $98,5 \%$ & $100 \%$ & $99,2 \%$ \\
\hline
\end{tabular}

Tabelle 6: Durchsetzung der DKB in den Silbenendrandpositionen 1500-1770

Die Ergebnisse, die sich beim ergänzenden Zugriff auf ausgewählte Einzeltexte gewinnen lassen, können das Verdikt einer graphematischen Anomie indes nicht bestätigen. Zum einen treten insbesondere ab dem zweiten Viertel des 16. Jahrhunderts, also außerhalb des von Ebert et al. skizzierten zeitlichen Rahmens, Texte auf, deren Usus die mittelhochdeutsche Normalverteilung 'Silbengelenk: DKB vs. Silbenendrand: Einzelbuchstabe' zeigt (KT 25 [Augsburg 1528], KT 26 [Nürnberg 1528], KT 27 [Nürnberg 1525], KT 28 [Wittenberg 1527], KT 29 [Leipzig 1529], KT 30 [Erfurt 1531], KT 31 [Mainz 1530], KT 34 [Mainz $1532]^{10}$ ). In geringerem Umfang finden sich derartige Texte noch bis Mitte des 17. Jahrhunderts (KT 93 [Wien 1650]).

$\mathrm{Zu}$ diesem Zeitpunkt ist zum anderen verstärkt eine Neuordnung der Verteilung Einzel/Doppelkonsonantenbuchstabe in den Silbenendrandpositionen zu beobachten, die zwar einer morphembezogenen Neuorientierung zuwiderläuft, deswegen aber keinesfalls als ungeregelt bezeichnet werden darf. Betrachtet man diesbezüglich etwa die Verteilung der DKB in den Silbenendrandpositionen in KT 97 [Straßburg 1650], lässt sich folgendes feststellen:

a) Ergibt sich ein Konsonantenbuchstabencluster, dessen Umfang <3 ist, so steht DKB

-bei sollen und wollen ausnahmslos:

<soll> (p. 14; p. 14; p. 28; p. 41; p. 45;p. 48; p. 50; p. 50; p. 52)

<will> (p. $9 ;$ p. 10; p. 13; p. 14; p. 21; p. 23; p. 26; p. 31; p. 35; p. 35; p. 37; p. 38; p. 38; p. $38 ;$ p. $39 ;$ p. 40; p. 40; p. 41; p. 41; p. 44; p. 45; p. 45; p. 45; p. 45; p. 45; p. 48; p. 48; p. 51; p. $51 ;$ p. $53 ;$ p. $53 ;$ p. $54 ;$ p. 54); <woll > (p. 21)

-bei können erst ansatzweise:

$<$ kan> (p. 9; p. 9; p. 16; p. 24; p. 37; p. 44; p. 44; p. 45; p. 51; p. 51; p. 51; p. 53; p. 53; p. 53; p. $54 ;$ p. 54) vs. $<$ kann $>$ (p. $19 ;$ p. $45 ;$ p. 51)

\footnotetext{
${ }^{10}$ Regionale Faktoren spielen offensichtlich keine Rolle: In dem wie KT 31 bei Johann Schöffer in Mainz gedruckten KT 33 [Mainz 1531] weist das Paradigma von sollen 98,65\% und das Paradigma von wollen 84,62\% DKB in den Silbenendrandpositionen auf.
} 
b) Ergibt sich ein Konsonantenbuchstabencluster, dessen Umfang $\geq 3$ ist, steht einfacher Konsonatenbuchstabe

-bei sollen und wollen überwiegend:

<solt> (p. 28; p. 49) vs. <sollt> (p. 16) -<solte> (p. 7; p. 20; p. 22; p. 24; p. 26; p. 28; p. 37; p. 39; p. 42; p. 44) vs. <sollte $>$ (p. 16; p. 55) - <Soltestu $>$ (p. 44) $-<$ soltet $>$ (p. 25) vs. $<$ solltet $>$ (p. 52) $-<$ solten $>$ (p. 8; p. 8; p. 8; p. 8; p. 9; p. 22; p. 27; p. 29; p. 47)

$<$ wilt $>$ (p. 51) $-<$ wiltu $>$ (p. 52) - <wolt > (p. 14) $-<$ wolten > (p. 10; p. 13; p. 55; p. 55; p. 55) vs. <wollten> (p. 27), <wolte> (p. 11; p. 11; p. 18; p. 24; p. 27; p. 33; p. 46; p. 46; p. 47; p. 49 ; p. $49 ;$ p. 49; p. 54 ; p. 54)

-bei können ausschließlich:

$<$ ko̊nt $>$ (p. 28; p. 49) - <konte> (p. 7; p. 32; p. 49; p. 49; p. 50) $-<$ kondte $>$ (p. 46) - <ko̊nte> (p. 11; p. 18; p. 27; p. 27; p. 35; p. 40) $-<$ ko̊ntet $>$ (p. 37) $-<$ ko̊nten $>$ (p. 17) $-<$ geko̊nt $>$ (p. 45)

Auf der Makroebene lässt sich dieser Trend bestätigen. Betrachtet man exemplarisch die Werte für die Durchsetzung von $<11 t>\mid<11 s t>11$ gegenüber $<11>$ bei wollen, so zeigt sich, dass die in KT 97 nachgewiesene Verteilungsregel die Durchsetzung von DKB noch bis weit in18. Jahrhundert behindert, sofern dadurch Konsonantenbuchstabencluster des Umfangs $\geq 3$ entstehen

\begin{tabular}{|l|l|l|}
\hline Zeitschnitt & $\begin{array}{l}\text { Durchsetzung } \\
<\mathrm{ll}>\end{array}$ & $\begin{array}{l}\text { Durchsetzung <llt>, } \\
<\mathrm{llst}>\end{array}$ \\
\hline 1500 & $41,7 \%$ & $1,8 \%$ \\
\hline 1530 & $41,7 \%$ & $3,7 \%$ \\
\hline 1560 & $43,7 \%$ & $0 \%$ \\
\hline 1590 & $34,8 \%$ & $0 \%$ \\
\hline 1620 & $40,6 \%$ & $2 \%$ \\
\hline 1650 & $41,3 \%$ & $4,9 \%$ \\
\hline 1680 & $57,3 \%$ & $2,2 \%$ \\
\hline 1710 & $88,7 \%$ & $21,7 \%$ \\
\hline 1740 & $100 \%$ & $30 \%$ \\
\hline 1770 & $100 \%$ & $100 \%$ \\
\hline
\end{tabular}

Tabelle 7: Konsonantenbuchstabencluster im Paradigma von wollen im Silbenendrand

Die erste explizit morphematische Interpretation der DKB findet sich nach ihrer Durchsetzung im Usus denn auch a. 1788 im Zusammenhang mit Adelungs Zurückweisung des Clusterkriterums (zit. nach P. Ewald 2004: 111), nachdem seine Vorgänger lediglich die Schwankungen in der Norm konstatiert hatten:

\footnotetext{
${ }^{11}$ Die analogiebedingte Einfügung eines dentalen Frikativs für die 2. Sg. Ind. Präs. tritt bei sollen im 2. Viertel des 16., bei wollen erst zu Beginn des 17. Jahrhunderts auf. Die Graphie <willst> ist in den Korpustexten dementsprechend erst spät, im letzten Drittel des 18. Jahrhunderts belegt.
} 
"Es ist daher eine sehr willkủrliche Behauptung, wenn einige Sprachlehrer wollen, daß, wenn ein verdoppelter identischer Consonant in der Conjugation einen anderen Consonanten nach sich bekommt, man einen der erstern wegwerfen kơnne, folglich du fålst, er fålt, ich solte, wolte, wůste, kůste u.s.f. für fållst, fållt, sollte, wollte, wůßte, kůßte."

Das Beispiel der DKB-Graphie zeigt, dass die Lautunabhängigkeit des Aufkommens einer Schreibung nicht unbedingt die Durchsetzung ihrer morphematischen Funktionalisierung begünstigen muss, sofern andere, im vorliegenden Fall aller Wahrscheinlichkeit nach ästhetische Faktoren ins Spiel kommen (cf. <Schifffahrt> vs. <Schiffahrt> in der aktuellen Reformdiskussion).

\section{Zur sprachhistorischen Einordnung der morphematischen Überformung der deutschen Orthographie}

Als einer jener Sprachlehrer, denen Adelung Willkür vorwirft, ließe sich J.G. Schottelius anführen, was aus moderner Perspektive umso überraschender erscheint, da dessen 'Ausführliche Arbeit Von der Teutschen HaubtSprache' (1663) am Bespiel der Doppelkonsonantenbuchstaben, aber eben unter Ausschluss von sollen, wollen und können, fundamentale Prinzipien der Stammwortschreibung entwickelt (cf. C. Moulin 2004: 67). Von besonderem Interesse ist dabei seine Begründung, die erstmals die Funktionalität morphembezogener Schreibungen im Sinne einer Profilierung der Erfassungsfunktion geschriebener Sprache expliziert:

Es irret auch ferner bey Lesung der Teutschen Sprache die Doppelung sothaner Stammletteren nicht allein gar nicht/ sondern es ist vielmehr dasselbe ein unfehlbares sonderliches Zeichen und Erkåntniß der Wurtzel/ oder des Stammwortes/ da man alsbald untrieglich abzunehmen hat/ daß/ wo solche zwey gedoppelte Buchstabe sich finden/ daselbst das Stammwort oder die Wurtzel des Wortes sey/ welches denen Auslånderen/ so Teutsch lernen wollen/ unschwer also zubeobachten/ und kein geringer Vortheil ist. Zum Exempel/ wañ man sagte: Unangestossen/ angestammte Güter/ abgelauffene Zeit/ etc. alhie kan man alsbald aus den gedoppelten Buchstaben $\mathrm{B} / \mathrm{mm} / \mathrm{ff} / \mathrm{abnehmen/} \mathrm{daß} \mathrm{daselbst} \mathrm{die} \mathrm{Wurtzel} \mathrm{oder} \mathrm{Stammwort/} \mathrm{nemlich} \mathrm{stoß/}$ Stamm/ lauff/ můsse verhanden seyn. (J. G. Schottelius 1663, 194, zit. nach C. Moulin 2004: 67)

Der Verweis auf den Zweitspracherwerb ("Auslånderen/ so Teutsch lernen wollen") ist eher als emblematisch aufzufassen und kann bei Bedarf auch für phonographische Normierungen eingeführt werden, wogegen sich für das Französische J.B. Bossuet 1673, also ein Jahrzehnt nach Schottelius, in den 'Observations sur l'orthographe de la langue francoise' wendet:

parce qu'en uolant instruire les étrangers et leur faciliter la prononciation de nostre langue, on la fait meconnoistre aux Francois mesmes. Si on écriuoit tans, chan, cham, emais ou émés, connaissais, anterreman, faisaiet, qui reconnoistroit ces mots? (Zit. nach B. Cerquiglini 1995 : 113),

um gleichzeitig unter Vorwegnahme des Wortüberlegenheitseffekts (cf. J.M. Cattell 1885) die bei Schottelius angedeutete Lesetheorie zu präzisieren:

On ne lit point lettre à lettre ; mais la figure entiere du mot fait son impression tout ensemble sur l'œil et sur l'esprit. 
Dass solche metasprachlichen Reflexionen in der zweiten Hälfte des 17. Jahrhunderts auftreten, ist vor dem Hintergrund des Zusammenspiels von Usus und Norm bei der morphematischen Überformung der deutschen Orthographie konsequent: Sie folgen zeitlich als dritter Schritt auf die normativ-morphematische Reinterpretation einer Phonographie nach ihrer Durchsetzung im Usus (graphischer Ausgleich der Plosivalternanzen) und die - wie gesehen mehr oder weniger erfolgreich, nach 1620 aber irreversible - normativmorphematische Reinterpretation einer Phonographie vor ihrer Durchsetzung (Umlautgraphie).

Hinsichtlich einer sprachhistorischen Erklärung von Aufkommen und Durchsetzung morphembezogener Schreibungen im Deutschen kursieren im Wesentlichen zwei Positionen, die sich vor dem Hintergrund des Zusammenspiels von Usus und Norm sowie der zeitgenössischen metasprachlichen Reflexion als komplementär erweisen. H. Günther (1988: 93) vermutet:

daß vor allem nicht-sprachliche Ursachen maßgeblich gewesen sind: Die mit der Entwicklung des Druckwesens verbundene Demotisierung der Schriftlichkeit und die Entwicklung einer Hoch-, National- oder eben "Schrift"sprache sind viel eher Kandidaten für die zentrale Ursache dieses Prozesses. Sie machten es notwendig, eine gleiche schriftliche Form für das gleiche Morphem in sehr verschiedenen Lautungen bereitzustellen.

Dieser Prozess betrifft nicht allein Schreibungen, die gegenwartssprachlich als morphemidentifizierend $\mathrm{zu}$ analysieren sind, sondern auf viel allgemeinerer Ebene die Herausbildung graphischer Morphemkonstanz, die sich historisch in erster Linie im Abbau von Schreibvarianten manifestiert. In den Texten des Untersuchungskorpus finden sich noch bis weit ins 16. Jahrhundert hinein extreme Schwankungen graphischer Wort- und Morphemformen. So zeigt eine systematische Betrachtung der Schreibungen von Kind in KT 50 [Köln 1557] graphische Varianz bei der Repräsentation von

Silbenkern:

$$
<\mathrm{i}>,<\mathrm{y}>
$$

Silbenendrand:

$<\mathrm{t}>,<\mathrm{dt}>,<\mathrm{d}>$

Von den sechs Graphiemöglichkeiten, die aus diesen Zuordnungsregeln ableitbar sind, werden immerhin fünf realisiert:

\begin{tabular}{|l|l|l|}
\hline & $<\mathrm{i}>$ & $<\mathrm{y}>$ \\
\hline$<\mathrm{t}>$ & $*<\mathrm{kint}>$ & $<$ kynt $>$ [f. A II ${ }^{\mathrm{r}}$ u.ö.] \\
\hline$<\mathrm{dt}>$ & $<$ kindt $>$ [f. B II ${ }^{\mathrm{r}}$ u.ö.] & $<$ kyndt $>$ [f. A II u.ö.] \\
\hline$<\mathrm{d}>$ & $<$ kind $>$ [f. B III ${ }^{\mathrm{v}}$ ] & $<$ kynd $>$ [f. A VI ${ }^{\mathrm{r}}$ ] \\
\hline
\end{tabular}

Tabelle 8: Schreibvarianten für nhd. $<$ Kind $>$ in KT 50

Bei näherer Betrachtung der variablen Elemente erweist sich die Variante $<y>$ statt $<\mathrm{i}>$ als regionale, genauer: alemannische und mittelfränkische Graphiebesonderheit (cf. V. Moser 1929: § 18). Die Variante $<\mathrm{d}>$ ist als Orientierung der Schreibung auf die mittelfränkisch lenisierte Flexionsform Kinder gegen die phonematisch orientierte Variante $<\mathrm{t}>$ interpretierbar (cf. V. Moser 1951: § 143,3a), die Variante <dt> als ein Kompromiss zwischen neuer morphematischer und alter phonematischer Orientierung. Die Variante $<\mathrm{z}>$ statt $<\mathrm{s}\rangle$ 
weist V. Moser (1929: § 45,2) nur bis Ende des 15. Jahrhunderts nach, ihr Auftreten für das germanische Genitiv-s ist besonders ungewöhnlich und spezifisch "md. und insbesondere mfr." (V. Moser 1951: § 146, 3c).

Abgesehen vom Abbau solcher Reflexe regionaler Schreibsprachen scheint neben der expliziten Profilierung der Erfassungsfunktion und damit des visuellen Kanals durch Bossuet und Schottelius sowie eher typo- als orthographischen Entwicklungen wie dem Abbau der einer rein visuellen Dekodierung hinderlichen determinativ-phonetischen Kürzungen Nasalstrich und er-Haken (cf. N. Ruge 2004: 207-232) v.a. die lautungsunabhängige

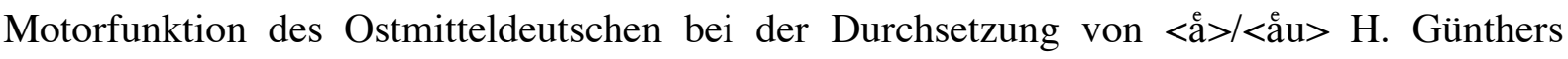
Einschätzung zu bestätigen.

Demgegenüber sieht P. Eisenberg (1983: 62f.) in Alphabetschriften grundsätzlich die Tendenz zur "Morphologisierung" angelegt. Die Orientierung der Schrift auf die Lautebene ist demnach zwar hinsichtlich des Zeicheninventars ökonomisch, der kontinuierliche Lautwandel führt aber dazu, dass mehr oder weniger reine Alphabetschriften in ihrer zwangsläufig diskreten Kodierungsweise ständig an Funktionalität zu verlieren drohen, da Schrift als eher fernesprachliches Medium auf Dauerhaftigkeit angewiesen ist. Die Orientierung der deutschen Alphabetschrift auf Morphemebene hat also sprachimmanente, strukturell-funktionale Gründe und wirkt nach P. Eisenberg (1983: 63) direkt auf die Hierarchie der Kodes: "An die Stelle der Lauttreue ist als Prinzip das Sichtbarmachen von morphologischen Zusammenhängen getreten." Abgesehen davon, dass "Lauttreue" - anders als etwa bei phonetischen Transkriptionssystemen - prinzipiell und im Fall des Deutschen (s.o.) schon gar kein funktionales Prinzip eines Graphiesystems sein kann, spricht für diese Position vor allem die frühzeitige Durchsetzung durch Substantivflexion ausgelöster $<\mathfrak{a}>/<$ åu $>$-Schreibungen sowie die Verzögerung der Durchsetzung von dentalem Lenisbuchstaben in Fällen, wo die Herstellung einer (in der Terminologie von Eisenberg prosodisch determinierten) Explizitform aus den verschiedensten Gründen Schwierigkeiten macht.

In der historischen Realität sind innersprachliche und außersprachlich Ursachen indes kaum voneinander $\mathrm{zu}$ trennen. Sicherlich begünstigt das Aufkommen des Buchdrucks Enkodierungsmodi, die sprachlandschaftsunabhängig funktionieren, andererseits schreibt sich deren Evolution in einen Kontext des Abrückens von der reinen Phonographie ein, der seit dem lateinischen Frühmittelalter im Gange ist. Zweitens ist bei der sprachhistorischen Untersuchung des Usus schwer $\mathrm{zu}$ bestimmen, welche Schreibung genau als morphembezogen $\mathrm{zu}$ qualifizieren ist, sobald man die Parameter möglicher Kommunikationssituationen durchspielt: Wenn beispielsweise oben davon ausgegangen

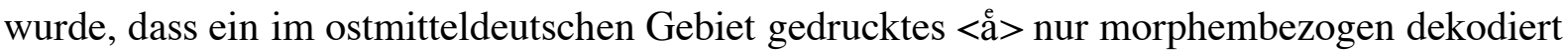
werden konnte, so gilt diese Einschätzung strenggenommen allein für einen Rezipienten ostmitteldeutscher Mundart, wobei zusätzlich der sprachsoziologische Wandel im Verhältnis von Lautung und Schreibung zu berücksichtigen wäre, der seit dem 15. Jahrhundert dazu führt, dass sich Schreibsprachen "weitgehend unabhängig von den gesprochenen Dialekten der betreffenden Region" (P. von Polenz 1991: 167) entwickelten.

Schließlich spricht gegen eine analytische Dissoziation inner- und außersprachlicher Faktoren, dass die metasprachliche Reflexion, namentlich Schottelius, Morphembezug ("ein 
unfehlbares sonderliches Zeichen und Erkăntniß der Wurtzel/ oder des Stammwortes") und mit Visualisierung funktional konnotierte 'Demotisierung' im weiteren Sinne ("welches denen Auslånderen/ so Teutsch lernen wollen/ unschwer also zubeobachten/ und kein geringer Vortheil ist") explizit verknüpft.

\section{Literaturangaben}

Bach, H[einrich] (1985): Handbuch der Luthersprache. Laut- und formenlehre in Luthers Wittenberger drucken bis 1545, II. Druckschwache silben. Konsonantismus. Kopenhagen.

Boková, Hildegard (1998): Der Schreibstand der deutschsprachigen Urkunden und Stadtbucheintragungen Südböhmens aus vorhussitischer Zeit (1300-1419). Frankfurt/Main etc.

Cattell, J. McKenna (1885): "Über die Zeit der Erkennung und Benennung von Schriftzeichen, Bildern und Farben". Philosophische Studien 2: 635-650.

Cerquiglini, Bernard (1995): L'accent du souvenir. Paris.

Dammers, Ulf/Hoffmann, Walter/Solms, Hans-Joachim (1988): Grammatik des Frühneuhochdeutschen. Beiträge zur Laut- und Formenlehre, hgg. von Hugo Moser Hugo Stopp - Werner Besch, IV. Flexion der starken und schwachen Verben. Heidelberg.

Eisenberg, Peter (1983): "Orthographie und Schriftsystem". In: Günther, Klaus B./Günther, Hartmut (eds.): Schrift, Schreiben, Schriftlichkeit. Arbeiten zur Struktur, Funktion und Entwicklung schriftlicher Sprache. Tübingen: 41-68.

Eisenberg, Peter (1996): "Zur Typologie der Alphabetschriften. Das Deutsche und die Reform seiner Orthographie". In: Lang, Ewald/Zifonun, Gisela (eds.): Deutsch - typologisch. Berlin - New York 1996: 615-631.

Ewald, Petra (2004): "Das morphematische Prinzip bei den Grammatikern des 18. Jahrhunderts". Sprachwissenschaft 29: 75-132.

Ebert, Robert Peter et al. (1993): Frühneuhochdeutsche Grammatik. Tübingen.

Glaser, Elvira (1985): Graphische Studien zum Schreibsprachwandel vom 13. zum 16. Jahrhundert. Vergleich verschiedener Handschriften des Augsburger Stadtbuches. Heidelberg.

Günther, Hartmut (1988): Schriftliche Sprache. Strukturen geschriebener Wörter und ihre Verarbeitung beim Lesen. Tübingen.

Henkel, Nikolaus (1996): "Die althochdeutschen Interlinearversionen. Zum sprach- und literaturhistorischen Zeugniswert einer Quellengruppe". In: Heinzle, Joachim/Johnson, L. Peter/Vollmann-Profe, Gisela (eds.): Übersetzen im Mittelalter. Cambridger Kolloquium 1994 (=Wolfram-Studien XIV). Berlin: 46-77.

Hotzenköcherle, Rudolf (1962): "Entwicklungsgeschichtliche Grundzüge des Neuhochdeutschen". Wirkendes Wort 12: 321-331.

Kohrt, Manfred (1991): "Deutsche 'Dentalepenthese' und 'Auslautverhärtung'. Phonologische, morphologische und orthographische Aspekte, sowohl synchron als auch diachron". In: Feldbusch, Elisabeth/Pogarell, Rainer/Weiß, Cornelia (eds.): Neue Fragen der Linguistik. Akten des 25. Linguistischen Kolloquiums Paderborn 1990. Band I., Tübingen: 531-537.

Kranzmayer, Eberhard (1956): Historische Lautgeographie des gesamtbairischen Dialektraumes mit 27 Laut- und 4 Hilfskarten in besonderer Mappe. Wien. 
Labs-Ehlert, Brigitte (1993): Versalschreibung in althochdeutschen Sprachdenkmälern. Ein Beitrag über die Anfänge der Großschreibung im Deutschen unter Berücksichtigung der Schriftgeschichte. Göppingen.

Mihm, Arend (2004): "Zur Geschichte der Auslautverhärtung und ihrer Erforschung". Sprachwissenschaft 29: 133-206.

Moser, Virgil (1929): Frühneuhochdeutsche Grammatik, I. Lautlehre, 1. Orthographie, Betonung, Stammsilbenvokale. Heidelberg.

Moser, Virgil (1951): Frühneuhochdeutsche Grammatik, I. Lautlehre, 3. Konsonanten, 2. Hälfte (Schluss). Heidelberg.

Moulin, Claudine (2004): "Das morphematische Prinzip bei den Grammatikern des 16. und 17. Jahrhunderts". Sprachwissenschaft 29: 33-73.

Nerius, Dieter et al (2000): Duden. Deutsche Orthographie. Mannheim etc.

Otto, Ernst (1970): Die Sprache der Zeitzer Kanzleien im 16. Jahrhundert. Untersuchungen zum Vokalismus und Konsonantismus. Berlin.

Parkes, Malcom Beckwith (1992): Pause and Effect. An Introduction to the History of Punctuation in the West. Cambridge.

Patzlaff, Rainer (1975): Otfrid von Weißenburg und die mittelalterliche versus-Tradition. Untersuchungen zur formgeschichtlichen Stellung der Otfridstrophe. Tübingen.

Paul, Hermann ( $\left.{ }^{24} 1998\right):$ Mittelhochdeutsche Grammatik. Überarbeitet von Peter Wiehl und Siegfried Grosse. Tübingen.

Polenz, Peter von (1991): Deutsche Sprachgeschichte vom Spätmittelalter bis zur Gegenwart, I. Einführung - Grundbegriffe - Deutsch in der frühbürgerlichen Zeit. Berlin/New York.

Ruge, Nikolaus (2004): Aufkommen und Durchsetzung morphembezogener Schreibungen im Deutschen 1500-1770. Heidelberg.

Saenger, Paul (1997): Space Between Words. The Origins of Silent Reading. Stanford.

Sampson, Geoffrey (1985): Writing Systems. A linguistic Introduction. London etc.

Simmler, Franz (1983): "Konsonantenschwächungen in den deutschen Dialekten". In: Besch, Werner et al. (eds.): Dialektologie. Ein Handbuch zu deutschen und allgemeinen Dialektforschung (=HSK. 1.2). Berlin/New York: 1121-1129.

Vaught, George Mason (1977): A study of Auslautverhärtung in Old High German. Ann Arbor.

Walch, Maria/Häckel, Susanne (1988): Grammatik des Frühneuhochdeutschen. Beiträge zur Laut- und Formenlehre, hgg. von Hugo Moser - Hugo Stopp - Werner Besch, VII. Flexion der Pronomina und Numeralia. Heidelberg.

Wegera, Klaus-Peter (1987): Grammatik des Frühneuhochdeutschen. Beiträge zur Laut- und Formenlehre, hgg. von Hugo Moser - Hugo Stopp - Werner Besch, III. Flexion der Substantive. Heidelberg.

Wiesinger, Peter (1996): Schreibung und Aussprache im älteren Frühneuhochdeutschen. Zum Verhältnis von Graphem - Phonem - Phon am bairisch-österreichischen Beispiel von Andreas Kurzmann um 1400. Berlin /New York (=Studia Linguistica Germanica 42). 